\title{
Theoretical Limits of Damping
}

\author{
Attainable by Smart Beams
}

\author{
with Rate Feedback
}

\author{
A.V. Balakrishnan \\ Flight Systems Research Center \\ UCLA
}

April 1997

To be published in: Proceedings of SPIE's 4th Annual Symposium on Smart Structures and Materials, 2-6 March 1997, San Diego, California. 


\title{
Theoretical Limits of Damping Attainable by Smart Beams with Rate Feedback
}

\author{
A.V. Balakrishnan \\ Flight Systems Research Center, UCLA
}

\begin{abstract}
Using a generally accepted model we present a comprehensive analysis (within the page limitation) of an EulerBernoulli beam with PZT sensor-actuator and pure rate feedback. The emphasis is on the root locus - the dependence of the attainable damping on the feedback gain. There is a critical value of the gain beyond which the damping decreases to zero. We construct the time-domain response using semigroup theory, and show that the eigenfunctions form a Riesz basis, leading to a "modal" expansion.
\end{abstract}

\section{INTRODUCTION}

In this paper we present a comprehensive analysis of an Euler-Bernoulli beam with PZT sensor-actuator along its entire length. The sensor output is a charge in a condenser and the actuator input is the current, a differentiator circuit being then an essential component, yielding "rate feedback." We use a generally accepted model. ${ }^{1-4}$ Tzou et al. ${ }^{4}$ present purely computational results and seem to be unaware of a purely theoretical analysis given earlier by Chen et al. ${ }^{5}$ The most important design parameter is the control gain and the damping attainable - we construct a full root-locus analysis (omitting details to keep within the page limitation). We also unearth a curious phenomenon - the existence of a deadbeat mode (real eigenvalue) not noticed hitherto. We show that the eigenvalues are the roots of an entire function of order one-half, proving in particular the existence of a countably infinite number of eigenvalues. We also show that the eigenfunctions form a Riesz basis. We also construct the Green's function for the nonhomogeneous eigenvalue problem. As in Chen et al. we use the theory of semigroups of operators to obtain the time-domain solution. Our proof of the exponential stability is different from that in Chen et al., as is our choice of the function space. We note that a similar analysis for a Timoshenko model (a "smart string") is given in Balakrishnan, ${ }^{6}$ where there is a critical value of the gain at which there are no eigenvalues and the semigroup is actually nilpotent ("disappearing" solution).

\section{MAIN RESULTS}

The Euler-Bernoulli model formulates as

$$
\left.\begin{array}{c}
c f^{\prime \prime \prime \prime}(t, s)+m \ddot{f}(t, s)=0, \quad 0<s<L, 0<t \\
f(t, 0)=0=f^{\prime}(t, 0) ; \quad f^{\prime \prime \prime}(t, L)=0 \\
c f^{\prime \prime}(t, L)+\alpha \dot{f}^{\prime}(t, L)=0
\end{array}\right\}
$$

where $f(t, s)$ is the displacement and the superdots indicate derivative with respect to $t$ and the primes indicate derivative with respect to $s$. It is convenient to set

$$
\nu^{2}=\frac{m}{c}
$$

For a precise formulation of the time-domain response we need to specify first the choice of function spaces. We pick $L_{2}[0, L]$ for $f(t, \cdot)$. Let $A_{o}$ denote the operator defined by

$$
A_{\circ} f=c f^{\prime \prime \prime \prime}
$$

where

$$
\mathcal{D}\left(A_{o}\right)=\left[f \mid f^{\prime}, f^{\prime \prime}, f^{\prime \prime \prime}, f^{\prime \prime \prime \prime} \in L_{2}[0, L] ; f(0)=0=f^{\prime}(0)=f^{\prime \prime \prime}(L)\right]
$$


Let

$$
\mathcal{H}=L_{2}[0, L] \times E^{1} .
$$

Define the operator $A$ with domain and range in $\mathcal{H}$ by:

$$
x=\left|\begin{array}{l}
f \\
b
\end{array}\right|, \quad A x=\left|\begin{array}{c}
A_{o} f \\
c f^{\prime \prime}(L)
\end{array}\right|
$$

with domain

$$
\mathcal{D}(A)=\left[\left|\begin{array}{l}
f \\
b
\end{array}\right|, f \in \mathcal{D}\left(A_{o}\right) \text { and } b=f^{\prime}(L)\right] .
$$

It is convenient to adopt the notation

$$
A_{b} x=c f^{\prime \prime}(L), \quad x \in \mathcal{D}(A)
$$

Then for $x$ in $\mathcal{D}(A)$ :

$$
\begin{aligned}
{[A x, x] } & =\int_{0}^{L} c f^{\prime \prime \prime \prime}(s) \overline{f(s)} d s+c f^{\prime \prime}(L) \overline{f^{\prime}(L)} \\
& =c \int_{0}^{L}\left|f^{\prime \prime}(s)\right|^{2} d s .
\end{aligned}
$$

It is readily seen that $A$ has dense domain and is self-adjoint and nonnegative definite, and has compact resolvent. Also zero is not an eigenvalue. Let $\sqrt{A}$ denote the positive square root. On the product space

$$
\mathcal{D}(\sqrt{A}) \times L_{2}[0, L]
$$

introduce the "energy" inner product

$$
\begin{gathered}
\left.\{Y, Z]_{E}=\left[\sqrt{A} y_{1}, \sqrt{A} z_{1}\right]+m \mid y_{2}, z_{2}\right] \\
Y=\left|\begin{array}{l}
y_{1} \\
y_{2}
\end{array}\right|, \quad Z=\left|\begin{array}{c}
z_{1} \\
z_{2}
\end{array}\right| . \\
\mathcal{D}(\sqrt{A})=\left[\left|\begin{array}{l}
f \\
b
\end{array}\right| \mid f^{\prime \prime} \in L_{2}[0, L] \text { and } b=f^{\prime}(L), f(0)=f^{\prime}(0)=0\right] .
\end{gathered}
$$

For $y_{1}$ in $\mathcal{D}(A)$, we see that

$$
[Y, Y]_{E}=\left[A y_{1}, y_{1}\right]+m\left[y_{2}, y_{2}\right] \sim \text { "energy" (potential + kinetic). }
$$

We denote the product space under this inner product by $\mathcal{K}_{E}$ and note that it is a Hilbert space. Let $\mathcal{A}$ denote the operator defined by:

$$
\mathcal{A} Y=\left|\begin{array}{c}
f_{2} \\
\frac{-c f_{1}^{\prime \prime}(L)}{\alpha} \\
\frac{-A_{0} f}{m}
\end{array}\right|, \quad Y=\left|\begin{array}{c}
x \\
f_{2}
\end{array}\right|=\left|\begin{array}{c}
f_{1}(\cdot) \\
f_{1}^{\prime}(L) \\
f_{2}(\cdot)
\end{array}\right|
$$

and

$$
\mathcal{D}(\mathcal{A})=|x=| \begin{gathered}
f_{1}(\cdot) \\
f_{1}^{\prime}(L)
\end{gathered}|\in \mathcal{D}(A)|, \quad\left|\begin{array}{c}
f_{2}(\cdot) \\
f_{1}^{\prime \prime}(L)
\end{array}\right| \in \mathcal{D}(\sqrt{A}) .
$$

Thus defined we can verify that

$$
\mathcal{D}(\mathcal{A})=\mathcal{D}\left(\mathcal{A}^{*}\right)
$$

and that $\mathcal{A}$ is dissipative:

$$
\frac{1}{2}\left[\left(\mathcal{A}+\mathcal{A}^{*}\right) Y, Y\right]=\operatorname{Re}[\mathcal{A} Y, Y]_{E}=\frac{-1}{a}\left\|A_{b} x\right\|^{2}=\frac{-1}{a} c^{2}\left|f_{1}^{\prime \prime}(L)\right|^{2} .
$$


It is readily verified that $\mathcal{A}$ has a compact resolvent and that $\mathcal{A}$ generates a $C_{0}$ contraction semigroup. With these definitions, the system (1) goes over into the abstract formulation:

$$
\dot{Y}(t)=\mathcal{A} Y(t)
$$

This choice of the function space is technically different from that in Chen et al. ${ }^{5}$

\section{Eigenvalues and eigenfunctions of $\mathcal{A}$}

Our primary interest is in the modal decomposition - the eigenvalues of $\mathcal{A}$ and the corresponding eigenfunctions. Or, equivalently, in the resolvent of $\mathcal{A}$. Let $\mathcal{R}(\lambda, \mathcal{A})$ denote the resolvent of $\mathcal{A}$. Let

$$
\mathcal{R}(\lambda, \mathcal{A}) Y=Z
$$

where

$$
Y=\left|\begin{array}{c}
h_{1} \\
b \\
h_{2}
\end{array}\right|
$$

Since $Z \in \mathcal{D}(\mathcal{A})$, we can write

$$
Z=\left|\begin{array}{c}
f_{1}(\cdot) \\
f_{1}^{\prime}(L) \\
f_{2}(\cdot)
\end{array}\right|
$$

and

$$
(\lambda I-\mathcal{A}) Z=Y
$$

yields

$$
\begin{gathered}
\lambda f_{1}-f_{2}=h_{1} \\
\lambda f_{2}+\frac{A_{o} f_{1}}{m}=h_{2} \\
\lambda f_{1}^{\prime}(L)+\frac{c f_{1}^{\prime \prime}(L)}{\alpha}=b .
\end{gathered}
$$

Hence

$$
\left.\begin{array}{rlr}
\lambda^{2} \nu^{2} f_{1}(s)+f_{1}^{\prime \prime \prime \prime}(s) & =\nu^{2}\left(h_{2}(s)+\lambda h_{1}(s)\right), & 0<s<L \\
\lambda \alpha f_{1}^{\prime}(L)+c f_{1}^{\prime \prime}(L) & =\alpha b \\
f_{1}(0)=0 & =f_{1}^{\prime}(0)=f_{1}^{\prime \prime \prime}(L) .
\end{array}\right\}
$$

\section{Eigenvalues}

First we consider the eigenvalue problem, setting

$$
h_{1}=0=h_{2} ; \quad b=0 .
$$

Let

$$
\gamma=\sqrt{\lambda \nu} e^{i \theta / 2} e^{i \pi / 4}, \quad \gamma^{4}=-\lambda^{2} \nu^{2}
$$

where

$$
\lambda=|\lambda| e^{i \theta} .
$$

Then the solution satisfying the conditions at zero yields:

$$
f_{1}(s)=a(\operatorname{Cosh} \gamma s-\operatorname{Cos} \gamma s)+b(\operatorname{Sinh} \gamma s-\operatorname{Sin} \gamma s), \quad 0<s<L .
$$

The constants $a$ and $b$ are then determined by the conditions at $L$ :

$a\left(\lambda \alpha \gamma(\operatorname{Sinh} \gamma L+\operatorname{Sin} \gamma L)+c \gamma^{2}(\operatorname{Cosh} \gamma L+\operatorname{Cos} \gamma L)\right)+b\left(\lambda \alpha \gamma(\operatorname{Cosh} \gamma L-\operatorname{Cos} \gamma L)+c \gamma^{2}(\operatorname{Sinh} \gamma L+\operatorname{Sin} \gamma L)\right)=0$

$$
a \gamma^{3}(\operatorname{Sinh} \gamma L-\operatorname{Sin} \gamma L)+b \gamma^{3}(\operatorname{Cosh} \gamma L+\operatorname{Cos} \gamma L)=0
$$


Let

$$
H(\lambda)=\left|\begin{array}{cc}
\lambda \alpha \gamma(\operatorname{Sinh} \gamma L+\operatorname{Sin} \gamma L) & \lambda \alpha \gamma(\operatorname{Cosh} \gamma L-\operatorname{Cos} \gamma L) \\
+c \gamma^{2}(\operatorname{Cosh} \gamma L+\operatorname{Cos} \gamma L) & +c \gamma^{2}(\operatorname{Sinh} \gamma L+\operatorname{Sin} \gamma L) \\
\gamma^{3}(\operatorname{Sinh} \gamma L-\operatorname{Sin} \gamma L) & \gamma^{3}(\operatorname{Cosh} \gamma L+\operatorname{Cos} \gamma L)
\end{array}\right|
$$

and

$$
D(\lambda)=\operatorname{Det} H(\lambda)
$$

Then

$$
\begin{aligned}
D(\lambda)= & \left(\gamma^{4}\right)[(\operatorname{Cosh} \gamma L+\operatorname{Cos} \gamma L)(\lambda \alpha(\operatorname{Sinh} \gamma L+\operatorname{Sin} \gamma L)+c \gamma(\operatorname{Cosh} \gamma L+\operatorname{Cos} \gamma L)) \\
& -(\operatorname{Sinh} \gamma L-\operatorname{Sin} \gamma L)(\lambda \alpha(\operatorname{Cosh} \gamma L-\operatorname{Cos} \gamma L)+c \gamma(\operatorname{Sinh} \gamma L+\operatorname{Sin} \gamma L))] \\
= & 2 \gamma^{4}[c \gamma(1+\operatorname{Cosh} \gamma L \operatorname{Cos} \gamma L)+\lambda \alpha(\operatorname{Sinh} \gamma L \operatorname{Cos} \gamma L+\operatorname{Cosh} \gamma L \operatorname{Sin} \gamma L)] .
\end{aligned}
$$

We note that zero is not an eigenvalue. The eigenvalues $\left\{\lambda_{k}\right\}$ are thus determined by the nonzero roots of

$$
c \gamma(1+\operatorname{Cosh} \gamma L \operatorname{Cos} \gamma L)+\lambda \alpha(\operatorname{Sinh} \gamma L \operatorname{Cos} \gamma L+\operatorname{Cosh} \gamma L \operatorname{Sin} \gamma L)=0
$$

Or, using

$$
\lambda=\frac{-i \gamma^{2}}{\nu}
$$

we have

$$
(1+\operatorname{Cosh} \gamma L \operatorname{Cos} \gamma L)-\frac{i \gamma \alpha}{\nu c}(\operatorname{Sinh} \gamma L \operatorname{Cos} \gamma L+\operatorname{Cosh} \gamma L \operatorname{Sin} \gamma L)=0
$$

\section{Theorem $2.1^{\dagger}$}

$\mathcal{A}$ has exactly one real-valued eigenvalue.

\section{Proof}

Setting $L=1$, and using $\alpha$ to denote $\frac{a}{c \nu}$, and expressing the trigonometric products in (6) as sums, we have

$$
\begin{aligned}
f= & 1+\operatorname{Cosh} \gamma \operatorname{Cos} \gamma-i \alpha \gamma(\operatorname{Sinh} \gamma \operatorname{Cos} \gamma+\operatorname{Cosh} \gamma \operatorname{Sin} \gamma) \\
f=1+ & \frac{1}{2}(\operatorname{Cos} \gamma(1+i)+\operatorname{Cos} \gamma(1-i)) \\
& \quad-i \alpha \gamma \frac{1}{2}[\operatorname{Sinh} \gamma(1+i)+\operatorname{Sin} \gamma(1+i)+\operatorname{Sinh} \gamma(1-i)+\operatorname{Sin} \gamma(1-i)] .
\end{aligned}
$$

Hence making the 1:1 transformation

$$
\gamma=x(i-1)
$$

we obtain

$$
f(\gamma)=g(x)=1+\frac{1}{2}(\operatorname{Cos} 2 x+\operatorname{Cosh} 2 x)-\alpha x(\operatorname{Sin} 2 x+\operatorname{Sinh} 2 x)
$$

yielding an equivalent expression for determining the eigenvalues. Note that $g(\cdot)$ is real-valued for real values of $x$. Further

$$
g(0)=2
$$

while, as $x \rightarrow \infty$, ( $x$ real $)$, we note that

$$
g(x) \rightarrow-\infty \text {. }
$$

Hence there is a positive real root. Denote it $x_{0}$. Then

$$
\lambda=-\gamma^{2} i=-x^{2}(i-1)^{2} i=-2 x^{2} .
$$

Hence

$$
\lambda_{0}=-2 x_{0}^{2}
$$

\footnotetext{
${ }^{\dagger}$ Due to J. Lin; private communication.
} 
is an eigenvalue. We note that $x_{1}$ is the ony real-valued root of $g(\cdot)$. Indeed, if there is a real-valued eigenvalue of $\mathcal{A}$, we must have, denoting it by $\lambda_{1}$,

$$
\lambda_{1}=-2 x_{1}^{2}
$$

and $x_{0}$ must be a root of $g(\cdot)$. Hence

$$
x_{0}=x_{1}
$$

Or, $\lambda_{0}$ is the only real-valued eigenvalue of $\mathcal{A}$.

We note that the corresponding eigenfunction is given by

$$
\phi_{1}(s)=\left(\operatorname{Cosh} \gamma_{0}-\operatorname{Cos} \gamma_{0}\right)\left(\operatorname{Cosh} \gamma_{0} s-\operatorname{Cos} \gamma_{0} s\right)-\left(\operatorname{Sinh} \gamma_{0}-\operatorname{Sin} \gamma_{0}\right)\left(\operatorname{Sinh} \gamma_{0} s-\operatorname{Sin} \gamma_{0} s\right)
$$

where

$$
\gamma_{0}=x_{0}(i-1), \quad \lambda=-2 x_{0}^{2}
$$

\section{Theorem 2.2 (Chen, et al. ${ }^{5}$ )}

Let $\left\{\lambda_{k}\right\}$ denote the eigenvalues, and assume that

$$
\left|\lambda_{k}\right| \rightarrow \infty
$$

Then

$$
\lim _{k} \operatorname{Re} \lambda_{k}=\frac{-c}{L \alpha}
$$

\section{Proof}

See Chen et al. ${ }^{5}$ for a proof.

The authors of Chen et al. however do not appear to offer a proof of the fact that the eigenvalues $\left\{\lambda_{k}\right\}$ are nonfinite in number. The fact that the resolvent is compact is not adequate to establish this; the compactness only assures that if nonfinite in number then $\left\{\lambda_{k}\right\}$ can be arranged so that

$$
\left|\lambda_{k+1}\right| \geq\left|\lambda_{k}\right|
$$

and

$$
\left|\lambda_{k}\right| \rightarrow \infty \text { as } k \rightarrow \infty
$$

For proving the fact that eigenvalues are denumerably infinite we can indicate a general technique.

\section{Theorem 2.3}

The eigenvalues $\left\{\lambda_{k}\right\}$ are denumerably infinite and such that

$$
\sum_{1}^{\infty}\left|\operatorname{Im}\left(\frac{1}{\lambda_{k}}\right)\right|<\infty \text {. }
$$

Proof

From (6) we see that for each $\alpha$, the eigenvalues are the zeros of the function

$$
d(\lambda)=(1+\operatorname{Cosh} \gamma L \operatorname{Cos} \gamma L)-i\left(\frac{\alpha}{c \nu}\right) \gamma(\operatorname{Sinh} \gamma L \operatorname{Cos} \gamma L+\operatorname{Cosh} \gamma L \operatorname{Sin} \gamma L) .
$$

As a power series expansion will show, this is an entire function of the complex variable $\lambda$. Moreover it is of exponential type, of order $\frac{1}{2}$, and of completely regular growth. Further we can calculate that

$$
h(\theta)=\varlimsup_{r \rightarrow \infty} \log \left|d\left(r e^{i \theta}\right)\right|=\sqrt{2} \max \left(\left|\operatorname{Sin} \frac{\theta}{2}\right|,\left|\operatorname{Cos} \frac{\theta}{2}\right|\right) .
$$


Let $n(r)$ denote the number of zeros of $d(\cdot)$ in the circle of radius $r$ centered at zero. Then by the theorem of R.P. Boas (see Levin ${ }^{7}$ ) we have:

$$
\lim _{r \rightarrow \infty} \frac{n(r)}{r^{1 / 2}}=\frac{1}{4 \pi} \int_{0}^{2 \pi} h(\theta) d \theta>0 .
$$

Hence

$$
\lim _{r \rightarrow \infty} n(r)=\infty,
$$

or, the number of zeros is not finite. Moreover the function is of class A (see Levin ${ }^{7}$ for the definition) since

$$
\sup _{R>0} \int_{0}^{R} \frac{\log |d(s) d(-s)|}{1+s^{2}} d s<M_{d}<\infty .
$$

The result (9) is a consequence. Q.E.D.

\section{Remark}

Applying Jensen's Theorem we have

$$
\frac{1}{2 \pi} \int_{0}^{2 \pi} \frac{d^{\prime}\left(r e^{i \theta}\right)}{d\left(r e^{i \theta}\right)} r e^{i \theta} d \theta=n(r)
$$

We can acutally compute this as a quick means of locating eigenvalues. There is a jump of 2 corresponding to each eigenvalue and its conjugate. This is shown in Figure 1 for

$$
\frac{\alpha}{L c \nu}=.01 \text {. }
$$

\section{Eigenfunctions}

The eigenfunction corresponding to the eigenvalue $\lambda_{k}$ is given by

$$
\boldsymbol{\Phi}_{k}=A_{k}\left|\begin{array}{c}
\phi_{k} \\
\phi_{k}^{\prime}(L) \\
\lambda_{k} \phi_{k}
\end{array}\right|
$$

where

$$
\phi_{k}(s)=c_{k}\left(\operatorname{Cosh} \gamma_{k} s-\operatorname{Cos} \gamma_{k} s\right)+d_{k}\left(\operatorname{Sinh} \gamma_{k} s-\operatorname{Sin} \gamma_{k} s\right)
$$

where

$$
H\left(\lambda_{k}\right)\left|\begin{array}{c}
c_{k} \\
d_{k}
\end{array}\right|=0
$$

or, we may take

$$
c_{k}=\left(\operatorname{Cosh} \gamma_{k} L-\operatorname{Cos} \gamma_{k} L\right) ; \quad d_{k}=-\left(\operatorname{Sinh} \gamma_{k} L-\operatorname{Sin} \gamma_{k} L\right)
$$

or,

$$
\phi_{k}(s)=A_{k}\left[\left(\operatorname{Cosh} \gamma_{k} L-\operatorname{Cos} \gamma_{k} L\right)\left(\operatorname{Cosh} \gamma_{k} s-\operatorname{Cos} \gamma_{k} s\right)-\left(\operatorname{Sinh} \gamma_{k} L-\operatorname{Sin} \gamma_{k} L\right)\left(\operatorname{Sinh} \gamma_{k} s-\operatorname{Sin} \gamma_{k} s\right)\right] .
$$

Correspondingly:

$$
\phi_{k}^{\prime}(L)=2 A_{k} \gamma_{k}\left(\operatorname{Cosh} \gamma_{k} L-\operatorname{Cos} \gamma_{k} L\right) \operatorname{Sin} \gamma_{k} L
$$

The coefficient $A_{k}$ may be chosen for appropriate normalization. For example we may make

$$
\left\|\Phi_{k}\right\|=1 .
$$

Note that $\bar{\lambda}_{k}$ is an eigenvalue of $\mathcal{A}^{*}$ and the corresponding eigenvector is:

$$
\Psi_{k}=B_{k}\left|\begin{array}{c}
\bar{\phi}_{k}(\cdot) \\
\bar{\phi}_{k}(L) \\
-\bar{\lambda}_{k} \bar{\phi}_{k}(\cdot)
\end{array}\right|
$$


where $B_{k}$ is again a "normalization" scalar. Note that

$$
\begin{aligned}
{\left[\Phi_{k}, \Psi_{k}\right]_{E} } & =\left(c \int_{0}^{L} \phi_{k}^{\prime \prime}(s)^{2} d s-m \lambda_{k}^{2} \int_{0}^{L} \phi_{k}(s)^{2} d s\right) A_{k} \bar{B}_{k} \\
& =4 c A_{k} \bar{B}_{k} \gamma_{k}^{4} c_{k} d_{k} \int_{0}^{L}\left(\operatorname{Cosh} \gamma_{n} s \operatorname{Cos} \gamma_{n} s+\operatorname{Sinh} \gamma_{n} s \operatorname{Sin} \gamma_{n} s\right) d s \\
& =4 c c_{k} d_{k} A_{k} \bar{B}_{k} \gamma_{k} \operatorname{Cosh} \gamma_{k} L \operatorname{Sin} \gamma_{k} L \\
& \neq 0 .
\end{aligned}
$$

In particular we may choose $A_{k}, B_{k}$ so that

$$
\left[\Phi_{k}, \Psi_{k}\right]_{E}=1
$$

Further using a result of Gohberg and Krein ${ }^{10}$ (we omit the details) we can establish that $\left\{\Phi_{k}, \Psi_{k}\right\}$ with the normalization (12) actually yield a Riesz basis for $\mathcal{H}_{E}$. In terms of this basis we have the ("modal") expansion for the solution of (2)

$$
Y(t)=\sum_{1}^{\infty} a_{k} e^{\lambda_{k} t} \Phi_{k}
$$

where

$$
a_{k}=\left[Y(0), \Psi_{k}\right]_{E}
$$

and as an easy byproduct, using (8), we see that the semigroup generated by $\mathcal{A}$ is exponentially stable (established in Chen et al. by different arguments).

\section{Root Locus}

Let us consider how the eigenvalues behave as the gain $\alpha$ is varied. For this purpose it is convenient to define

$$
d(\lambda ; \alpha)=M(\lambda)+\frac{\alpha}{c \nu} N(\lambda)
$$

where

$$
\begin{gathered}
M(\lambda)=1+\operatorname{Cosh} \gamma L \operatorname{Cos} \gamma L \\
N(\lambda)=\frac{-i \gamma}{c \nu}(\operatorname{Sinh} \gamma L \operatorname{Cos} \gamma L+\operatorname{Cosh} \gamma L \operatorname{Sin} \gamma L) .
\end{gathered}
$$

Because of the analytic dependence of $d(\lambda ; \alpha)$ on $\alpha$, we can invoke the theory of algebraic or algebroidal functions 8,9 and note that

$$
d(\lambda(\alpha) ; \alpha)=0
$$

will define $\lambda(\alpha)$ as a multivalued analytic function of $\alpha$ with isolated singularities, if any. In particular this allows us to define the sequence $\left\{\lambda_{k}(\alpha)\right\}, k=1,2, \ldots$ such that

$$
\lambda_{k}(0)=\frac{i \mu_{k}^{2}}{L^{2} \nu}, \quad \mu_{k}=(2 k-1) \frac{\pi}{2}+\varepsilon_{k}
$$

(the "clamped-free" beam modes) and

$$
\lim _{\alpha \rightarrow \infty} \lambda_{k}(\alpha)=\frac{i\left(k \pi-\varepsilon^{\prime} k\right)^{2}}{L^{2} \nu}
$$

("clamped-rolling" modes) and the real root

$$
\lambda_{0}(\alpha)
$$

is such that

$$
\lim _{\alpha \rightarrow \infty} \lambda_{0}(\alpha)=0, \quad \lim _{\alpha \rightarrow 0} \lambda_{0}(\alpha)=-\infty
$$

A plot of the locus of the real root is shown in Figure 2. Moreover

$$
\lambda_{k}^{\prime}(\alpha)=\left.\frac{-1}{c \nu} \frac{N(\lambda)}{M^{\prime}(\lambda)+\frac{\alpha}{c \nu} N^{\prime}(\lambda)}\right|_{\lambda=\lambda_{k}(\alpha)} .
$$


In particular

$$
\lambda_{k}^{\prime}(0)=\left.\frac{-1}{c \nu} \frac{N(\lambda)}{M^{\prime}(\lambda)}\right|_{\lambda=\lambda_{k}(0)}
$$

We can show that

$$
\begin{array}{rlrl}
\frac{d}{d \alpha}\left(\operatorname{Re} \lambda_{k}(\alpha)\right) & =\frac{-\mu_{k}^{2}}{L^{2} \nu}\left(\frac{2}{L c \nu}\right), & & \alpha=0 \\
& =\frac{c}{2 \alpha^{2} L}, & & \alpha=+\infty \\
\frac{d}{d \alpha}\left(\operatorname{Im} \lambda_{k}(\alpha)\right) \geq 0 . & &
\end{array}
$$

A root locus of the first mode is shown in Figure 3. The damping $\left(=\left|\operatorname{Re} \lambda_{k}\right|\right)$ increases with the gain until a critical value of the gain is reached and thereafter decreases to zero. Note that by virtue of (14) we have actually "proportional damping" for small gain. A plot of the critical value of the gain versus the mode number is given in Figure 4.

\section{Resolvent}

Let us now return to the resolvent - or solving (3). We note that

$$
g(\lambda, s)=\frac{1}{2 \gamma^{3}} \int_{0}^{s}(\operatorname{Sinh} \gamma(s-\sigma)-\operatorname{Sin} \gamma(s-\sigma)) \nu^{2}\left(h_{2}(\sigma)+\lambda h_{1}(\sigma)\right) d \sigma
$$

is a "particular" solution of

$$
\lambda^{2} \nu^{2} f_{1}+f_{1}^{\prime \prime \prime}=\nu^{2}\left(h_{2}+\lambda h_{1}\right)
$$

such that

$$
f_{1}(0)=f_{1}^{\prime}(0)=0 \text {. }
$$

Hence we can express the solution $f_{1}(\lambda, s)$, where we have included $\lambda$ to indicate the dependence on $\lambda$, as:

$$
f_{1}(\lambda, s)=g(\lambda, s)+a(\lambda)(\operatorname{Cosh} \gamma s-\operatorname{Cos} \gamma s)+b(\lambda)(\operatorname{Sinh} \gamma s-\operatorname{Sin} \gamma s), \quad 0<s<L
$$

where the coefficients $a(\lambda), b(\lambda)$ are determined from

$$
\left|\begin{array}{l}
a(\lambda) \\
b(\lambda)
\end{array}\right|=H(\lambda)^{-1}\left|\begin{array}{c}
\alpha b-\alpha \lambda g^{\prime}(\lambda, L)-c g^{\prime \prime}(\lambda, L) \\
-g^{\prime \prime \prime}(\lambda, L)
\end{array}\right|
$$

where the primes again denote derivatives with respect to the variable s. Hence letting

$$
H(\lambda)=\left|\begin{array}{ll}
h_{11}(\lambda) & h_{12}(\lambda) \\
h_{21}(\lambda) & h_{22}(\lambda)
\end{array}\right|
$$

and defining

$$
\tilde{H}(\lambda)=\left|\begin{array}{rr}
h_{22}(\lambda) & -h_{12}(\lambda) \\
-h_{21}(\lambda) & h_{11}(\lambda)
\end{array}\right|
$$

so that

$$
\begin{gathered}
H(\lambda) \bar{H}(\lambda)=D(\lambda) I=\bar{H}(\lambda) H(\lambda) \\
a(\lambda)=\frac{1}{D(\lambda)}\left[h_{22}(\lambda)\left(\alpha b-\alpha \lambda g^{\prime}(\lambda, L)-c g^{\prime \prime}(\lambda, L)\right)+h_{12}(\lambda) g^{\prime \prime \prime}(\lambda, L)\right] \\
b(\lambda)=\frac{1}{D(\lambda)}\left[-h_{21}(\lambda)\left(\alpha b-\alpha \lambda g^{\prime}(\lambda, L)-c g^{\prime \prime}(\lambda, L)\right)-h_{11}(\lambda) g^{\prime \prime \prime}(\lambda, L)\right] .
\end{gathered}
$$


We can cast the Green's function in the form:

$$
\begin{gathered}
f_{1}(\lambda, s)=\int_{0}^{s} \frac{K(\lambda ; s, \sigma)}{D(\lambda)} h(\sigma) d \sigma+\frac{\int_{s}^{L} K(\lambda ; \sigma, s)}{D(\lambda)} h(\sigma) d \sigma \\
\left.+\frac{\alpha b}{D(\lambda)} \mid h_{22}(\lambda)(\operatorname{Cosh} \gamma s-\operatorname{Cos} \gamma s)-h_{21}(\lambda)(\operatorname{Sinh} \gamma s-\operatorname{Sin} \gamma s)\right] \\
K(\lambda ; \sigma, s)=(\operatorname{Cosh} \gamma s-\operatorname{Cos} \gamma s)\left[\left(2 h_{12}-\frac{2 \alpha \lambda}{\gamma^{2}} h_{22}\right) \operatorname{Cosh} \gamma(L-\sigma)+\left(2 h_{12}+\frac{2 \alpha \lambda}{\gamma^{2}} h_{22}\right) \operatorname{Cos} \gamma(L-\sigma)\right. \\
\left.-\frac{2 c h_{22}}{\gamma}(\operatorname{Sinh} \gamma(L-\sigma)+\operatorname{Sin} \gamma(L-\sigma))\right] \\
+(\operatorname{Sinh} \gamma s-\operatorname{Sin} \gamma s)\left[\left(\frac{2 \alpha \lambda h_{21}}{\gamma^{2}}-2 h_{11}\right) \operatorname{Cosh} \gamma(L-\sigma)+\left(\frac{-2 \alpha \lambda h_{21}}{\gamma^{2}}-2 h_{11}\right) \operatorname{Cos} \gamma(L-\sigma)\right. \\
\left.+\frac{2 c h_{21}}{\gamma}(\operatorname{Sinh} \gamma(L-\sigma)+\operatorname{Sin} \gamma(L-\sigma))\right], \quad s<\sigma \\
h=m\left(h_{2}+\lambda h_{1}\right) \\
D(\lambda)=-2 \lambda^{2} \nu^{2}[c \gamma(1+\operatorname{Cosh} \gamma L \operatorname{Cos} \gamma L)+\lambda \alpha(\operatorname{Sinh} \gamma L \operatorname{Cos} \gamma L+\operatorname{Cosh} \gamma L \operatorname{Sin} \gamma L)] .
\end{gathered}
$$

Finally

$$
\mathcal{R}(\lambda, \mathcal{A})\left|\begin{array}{c}
h_{1} \\
b \\
h_{2}
\end{array}\right|=\left|\begin{array}{c}
f_{1}(\lambda, s) \\
f_{1}^{\prime}(\lambda, 0) \\
\lambda f_{1}(\lambda, s)-h_{1}(s)
\end{array}\right|
$$

Note that setting $\alpha=0$ in (15) we get the Green's function for the clamped/free-free beam. In particular

$$
\mathcal{R}(0, \mathcal{A})\left|\begin{array}{c}
h_{1} \\
h_{1}(0) \\
h_{2}
\end{array}\right|=\left|\begin{array}{c}
K h_{2} \\
\left(K h_{2}\right)(0) \\
-h_{1}
\end{array}\right|+\alpha h_{1}(0)\left|\begin{array}{c}
\frac{L-s}{c} \\
\frac{L}{c} \\
0
\end{array}\right|
$$

where $K h_{2}$ is the function given by

$$
\frac{m}{c} \int_{0}^{s}(L-\sigma) h_{2}(\sigma) d \sigma+\frac{m}{c} \int_{L}^{s}(L-\sigma) h_{2}(\sigma) d \sigma, \quad 0<s<L .
$$

\section{ACKNOWLEDGEMENTS}

Research supported in part under grant NCC 2-374, NASA. I am indebted to J. Lin for the graphs.

\section{REFERENCES}

1. T. Bailey and J.E. Hubbard, Jr. "Distributed Piezoelectric-Polymer Active Vibration Control of a Cantilever Beam," J. Guidance, 8:5, pp. 605-611, 1985.

2. C.-K. Lee, W.-W. Chiang and T.C. O'Sullivan. "Piezoelectric Modal Sensor/Actuator Pairs for Critical Active Damping Vibration Control," J. Acoust. Soc. Am., 90:1, pp. 394-384, 1991.

3. H.S. Tzou and M. Gadre. "Theoretical Analysis of a Multi-layered Thin Shell Coupled with Piezoelectric Shell Actuators for Distributed Vibration Controls," J. Sound and Vibration, 132:3, pp. 433-450, 1989. 
4. H.S. Tzou, D.D. Johnson and K.J. Liu. "Nonlinear Control and Boundary Transition of Cantilevered Distributed Systems." In: Wave Motion, Intelligent Structures and Nonlinear Mechanics, edited by A. Guran and D.J. Inman, pp. 163-193. Series on Stability, Vibration and Control of Structures, Vol. 1. World Scientific Publishing Company, 1995.

5. G. Chen, S.G. Krantz, D.W. Ma, C.E. Wayne and H.H. West. "The Euler-Bernoulli Beam Equation with Boundary Energy Dissipation." In: Operator Methods for Optimal Control Problems, pp. 67-96. Lecture Notes in Mathematics, Vol. 108. Marcel Dekker, 1987.

6. A.V. Balakrishnan. "Vibrating Systems with Singular Mass-Inertia Matrices." In: Proceedings of First International Conference on Nonlinear Problems in Aviation \& Aerospace, Dayton Beach. Florida. 9-11 May 1996, pp. 23-32.

7. B.Ja. Levin. Distribution of Zeros of Entire Functions. American Math Society, 1980.

8. T. Kato. Perturbation Theory for Linear Operators. Springer-Verlag, 1976.

9. K. Knopp. Theory of Functions, Part 2. Dover Publications, 1947.

10. I.C. Gohberg and M.G. Krein. Introduction to the Theory of Linear Non-Selfadjoint Operators. Translations of Mathematical Monographs, Vol. 18. American Mathematical Society, 1969.

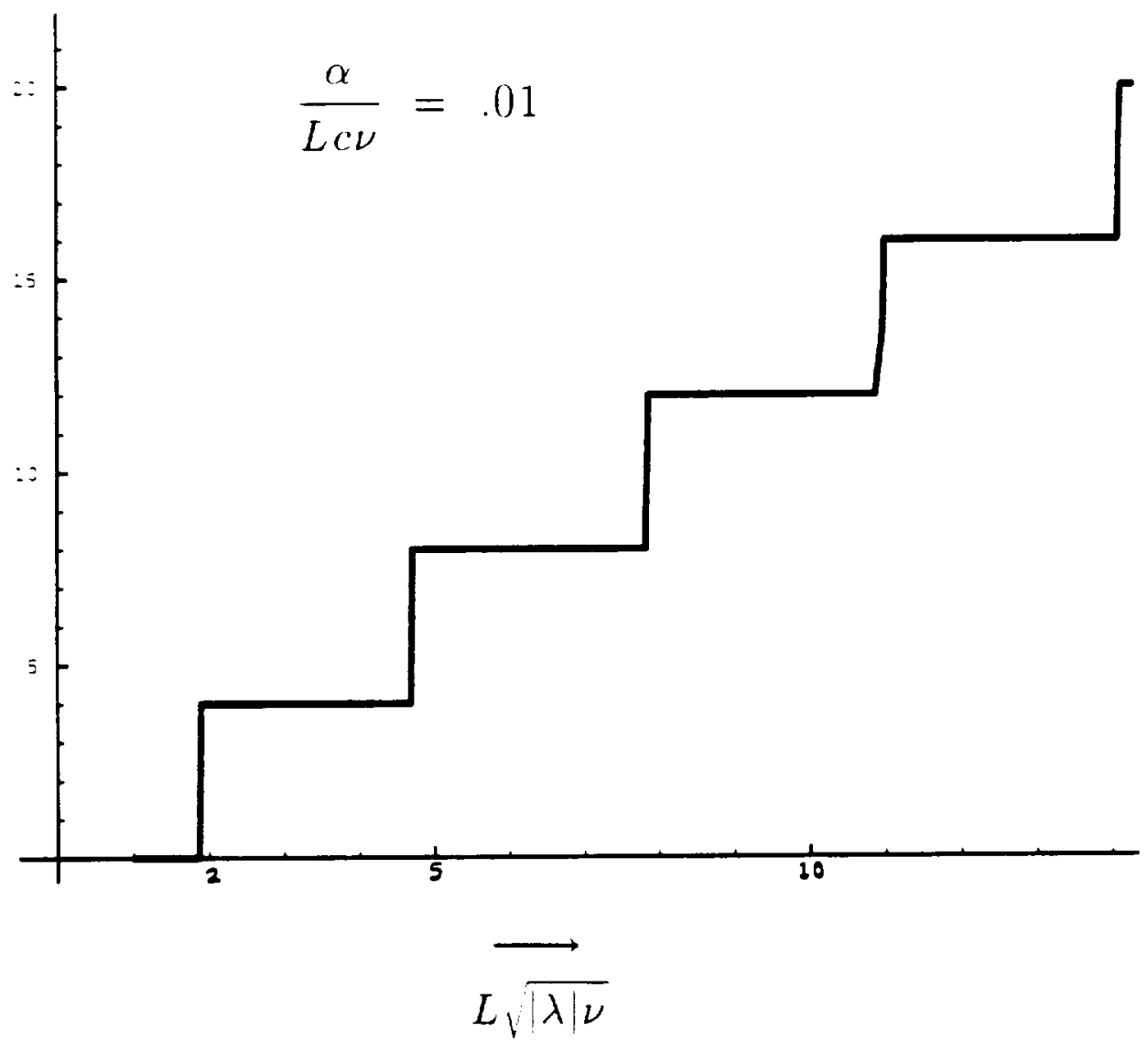

Figure 1: $n(|\lambda|)$ Zeros. 


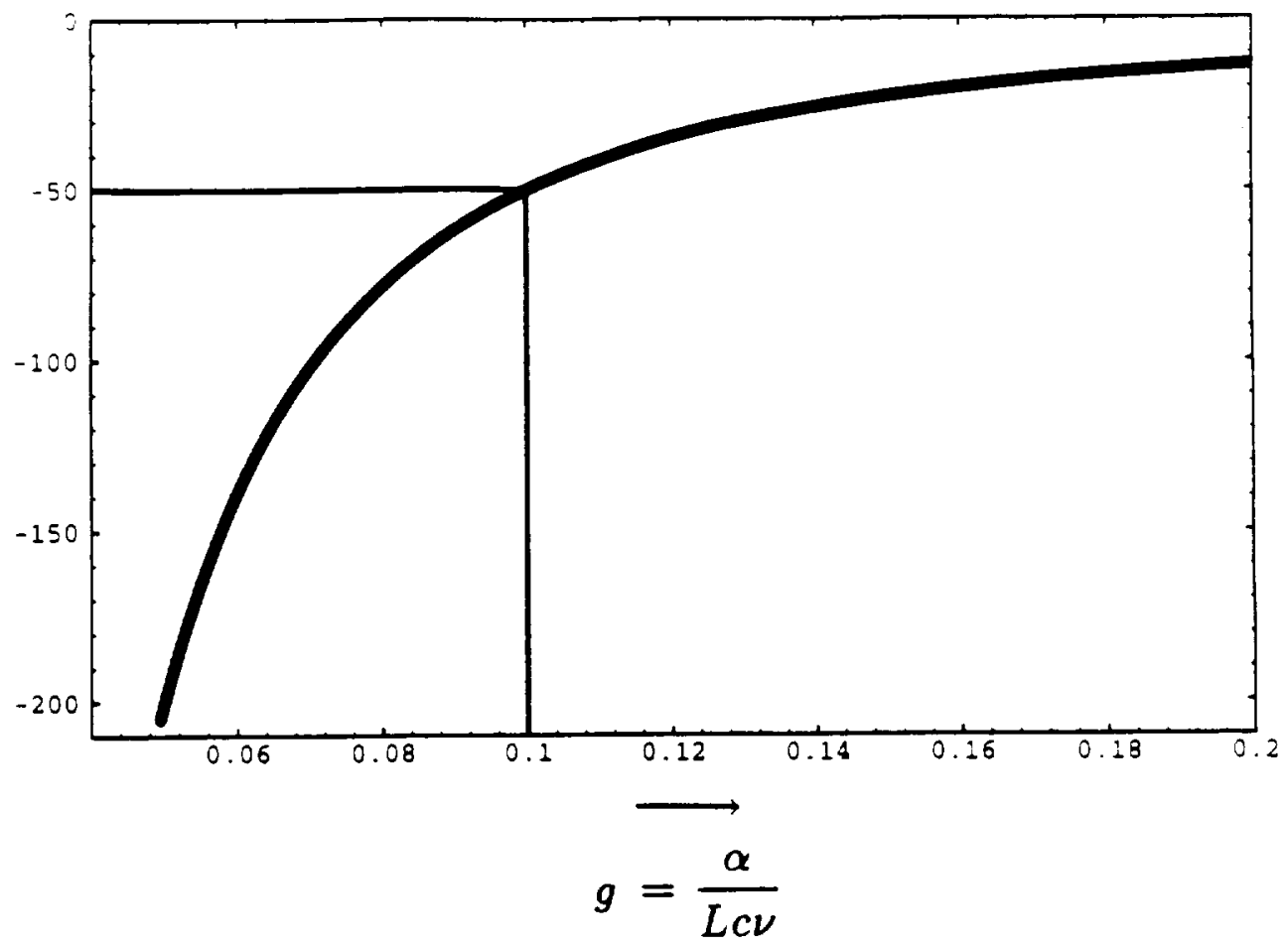

Figure 2: Deadbeat Mode (Real Eigenvalue).

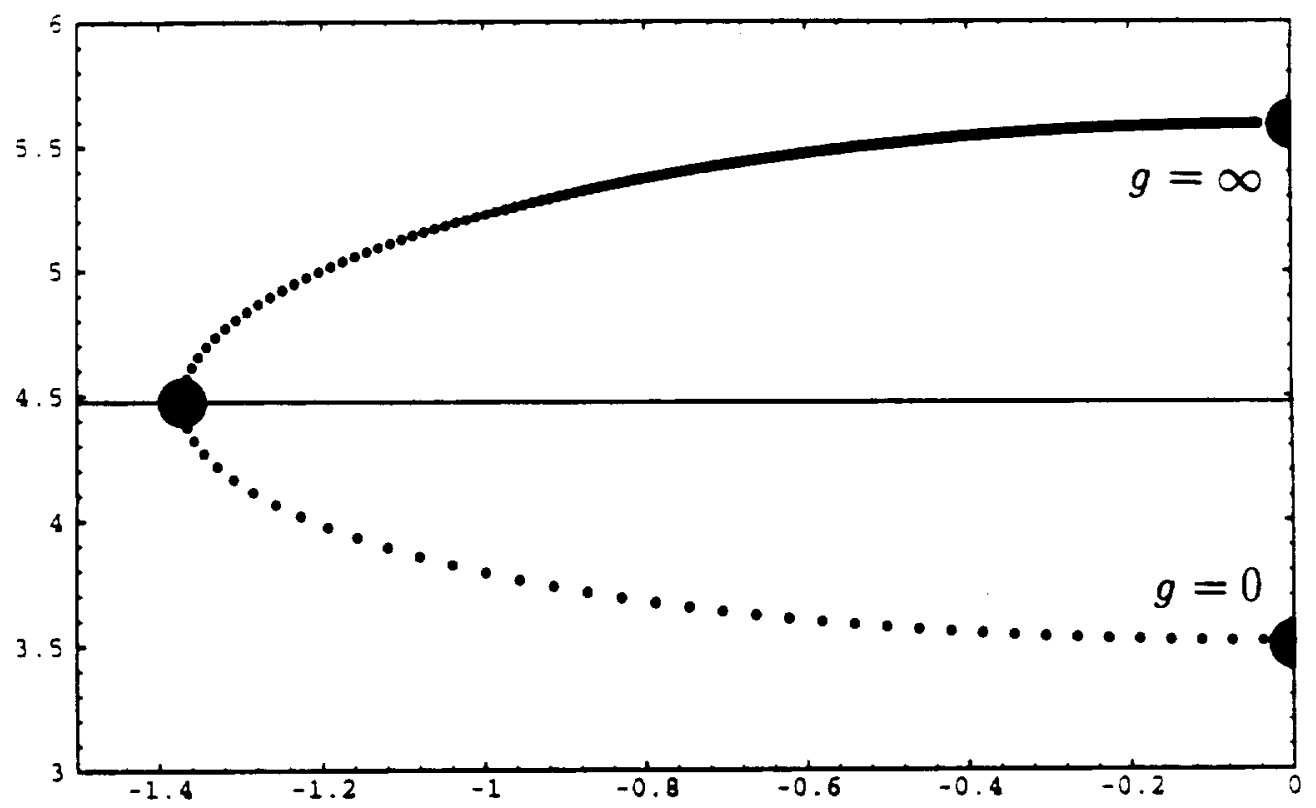

Figure 3: Root Locus: First Mode $\lambda=\sigma+i \omega$. 


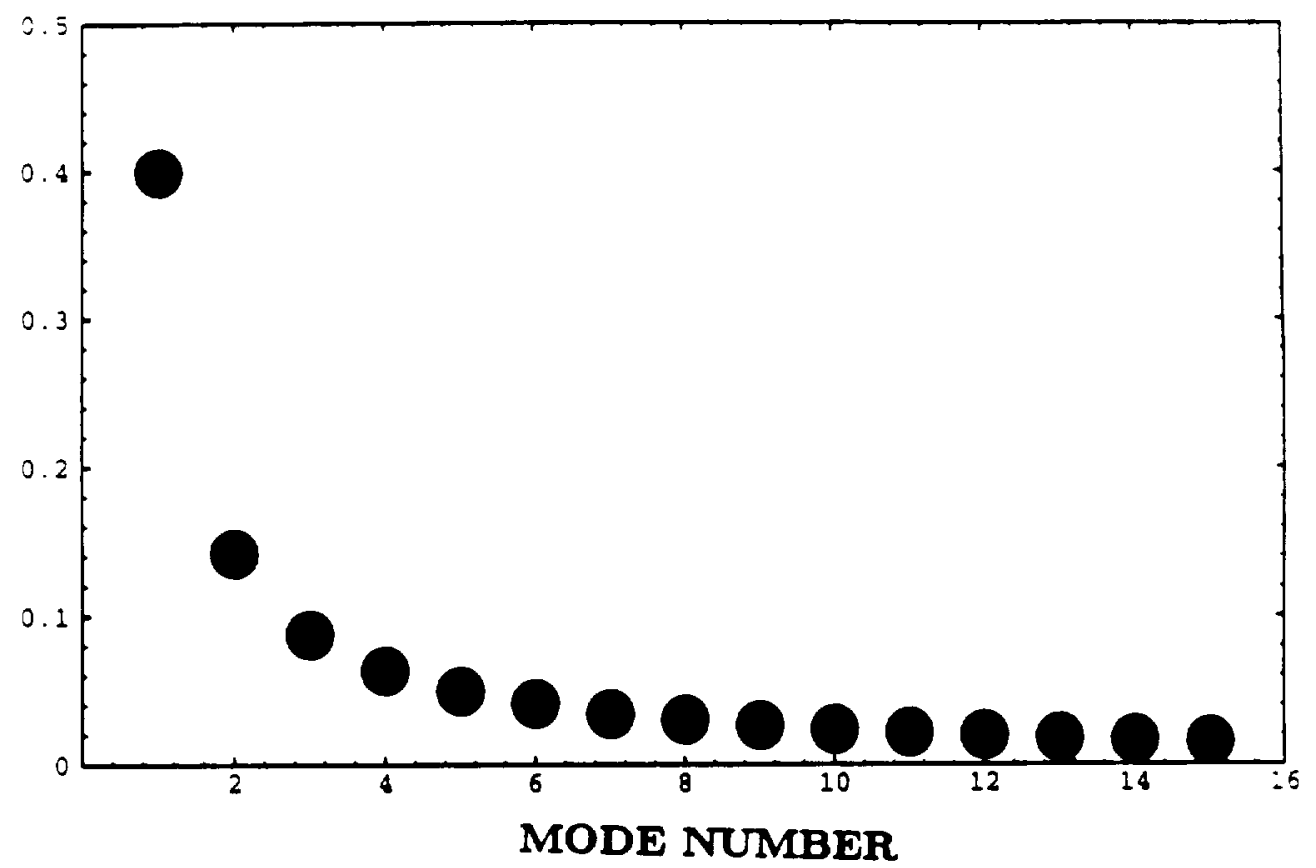

Figure 4: Critical Gain vs. Mode. 